\title{
Significance tests for the wavelet cross spectrum and wavelet linear coherence
}

\author{
Z. Ge \\ Ecosystems Research Division, NERL, USEPA, 960 College Station Road, Athens, GA 30605, USA
}

Received: 9 July 2008 - Accepted: 21 October 2008 - Published: 2 December 2008

\begin{abstract}
This work attempts to develop significance tests for the wavelet cross spectrum and the wavelet linear coherence as a follow-up study on Ge (2007). Conventional approaches that are used by Torrence and Compo (1998) based on stationary background noise time series were used here in estimating the sampling distributions of the wavelet cross spectrum and the wavelet linear coherence. The sampling distributions are then used for establishing significance levels for these two wavelet-based quantities. In addition to these two wavelet quantities, properties of the phase angle of the wavelet cross spectrum of, or the phase difference between, two Gaussian white noise series are discussed. It is found that the tangent of the principal part of the phase angle approximately has a standard Cauchy distribution and the phase angle is uniformly distributed, which makes it impossible to establish significance levels for the phase angle. The simulated signals clearly show that, when there is no linear relation between the two analysed signals, the phase angle disperses into the entire range of $[-\pi, \pi]$ with fairly high probabilities for values close to $\pm \pi$ to occur. Conversely, when linear relations are present, the phase angle of the wavelet cross spectrum settles around an associated value with considerably reduced fluctuations. When two signals are linearly coupled, their wavelet linear coherence will attain values close to one. The significance test of the wavelet linear coherence can therefore be used to complement the inspection of the phase angle of the wavelet cross spectrum.
\end{abstract}

The developed significance tests are also applied to actual data sets, simultaneously recorded wind speed and wave elevation series measured from a NOAA buoy on Lake Michigan. Significance levels of the wavelet cross spectrum and the wavelet linear coherence between the winds and the waves reasonably separated meaningful peaks from those generated by randomness in the data set. As with simu-

Correspondence to: $\mathrm{Z}$. Ge

(gezhfu@yahoo.com) lated signals, nearly constant phase angles of the wavelet cross spectrum are found to coincide with large values in the wavelet linear coherence between the winds and the waves. Not limited to geophysics, the significance tests developed in the present work can also be applied to many other quantitative studies using the continuous wavelet transform.

Keywords. Oceanography: physical (Air-sea interactions; Surface waves and tides) - General or miscellaneous (Techniques applicable in three or more fields)

\section{Introduction}

Ge (2007) revisited the issue of the significance tests for the wavelet power and the wavelet power spectrum. Torrence and Compo (1998) (referred to TC98 hereafter) are probably the first to have developed methods for these significance tests. They used background noise series for establishing the sampling distributions and the associated significance levels for interested wavelet-based quantities, which is essentially a conventional approach. Their methods and results have been adopted by many researchers (e.g. Torrence and Webster, 1999; Grinsted et al., 2004; Lundstedt et al., 2006; Rigozo et al., 2008) in the past ten years, especially in the fields of geophysics and engineering. Ge (2007) obtained analytical results of the sampling distributions of the wavelet power and wavelet power spectrum using prescribed background noise series. That was an improvement on TC98's results a large portion of which was based on Monte Carlo simulations. The present work was aimed to be a sequel to $\mathrm{Ge}$ (2007), continuing to develop significance tests for two other frequently used quantities (statistics), the wavelet cross spectrum and the wavelet linear coherence. Involving two time series instead of only one, these two wavelet-based quantities are often used to assess time-varying spectral relations between two signals, which are often nonstationary.

Published by Copernicus Publications on behalf of the European Geosciences Union. 
Perhaps the first one in geophysics, Liu (1994) defined the wavelet cross spectrum (WCS) between two time series $x(t)$ and $y(t)$ as

$C_{x y}(a, b)=X^{*}(a, b) Y(a, b)$,

where $a$ and $b$ are scale and time variables, respectively, $X(a, b)$ and $Y(a, b)$ are the wavelet coefficients of the time series $x(t)$ and $y(t)$, respectively, and $*$ means complex conjugate. In the definitions in Liu (1994) and TC98, the complex conjugate of $Y(a, b)$, instead of that of $X(a, b)$, was used. This inconsistency in definition will not affect any discussions below. Equation (1) has defined a WCS for each point in the time-scale domain, similar to the wavelet power. Since the WCS $C_{x y}$ is complex when complex wavelets such as the Morlet wavelet are used, its squared absolute value,

$\left|C_{x y}(a, b)\right|^{2}=\left|X^{*}(a, b) Y(a, b)\right|^{2}=|X(a, b)|^{2}|Y(a, b)|^{2}$,

or simply its absolute value $\left|C_{x y}(a, b)\right|$, is often plotted for visualisation (TC98). Apparently, a large value of $\left|C_{x y}(a, b)\right|^{2}$ occurs when the two signals $x$ and $y$ have large power at similar scales (frequencies) and around the same time, regardless of the local phase difference. When the phase information is desired, one needs to refer back to Eq. (1). Specifically, if the complex number $C_{x y}$ is expressed in terms of its module and phase angle, we have

$$
\begin{aligned}
C_{x y}(a, b) & =|X(a, b)| e^{-i \theta_{x}(a, b)}|Y(a, b)| e^{i \theta_{y}(a, b)} \\
& =\left|C_{x y}\right| e^{i\left(\theta_{y}(a, b)-\theta_{x}(a, b)\right)} .
\end{aligned}
$$

This means that the phase angle of the WCS, i.e. $\theta_{y}(a, b)-\theta_{x}(a, b)$, reflects the phase difference by which $y(t)$ leads $x(t)$ at the given scale and time. To present the information in phase Liu (1994) suggested that both the real and imaginary parts of the WCS be shown. Alternatively, another common practice is to show both the absolute value and the phase angle of $C_{x y}$ in the time-scale domain.

A well-known disadvantage of the WCS in the time-scale domain is that it cannot be normalised to have a value bounded by, for example, zero and one. A normalisation similar to what brings about the correlation coefficient between two time series always yields a value of unity for the WCS (TC98). In other words, the normalisation of the WCS cannot be done locally, and it must involve multiple points in the time-scale domain for some degree of smoothing. In geophysics as well as other physical fields, a simple averaging in time will yield a useful measure of the local linear coupling in the scale (frequency) domain between two signals. This averaged quantity is referred to as the wavelet linear coherence (WLC) (or sometimes the wavelet coherency) expressed as

$L_{x y}(a)=\frac{\left|\int_{T} X^{*}(a, b) Y(a, b) d b\right|^{2}}{\int_{T}|X(a, b)|^{2} d b \int_{T}|Y(a, b)|^{2} d b}$,

where $T$ is the time interval for averaging. $L_{x y}$ is zero for cases of no linear relation, is one for a perfect linear coupling, and has values between zero and one for linear relations of different degrees. It is important to note that the linear relation here is not exactly the same as the conditions that result in a large value in the $\left|C_{x y}\right|$. A large $L_{x y}$ at a particular scale $a$ results from persistently large wavelet energy densities at $a$ in both time series and a nearly constant phase difference between these two series at $a$ over a period $T$. The numerator on the right side of Eq. (4) will otherwise have a near zero value. The role that the phase difference plays in the WLC will become clearer in Sect. 4.

The significance test, one of the central ideas in statistical inference, is also important for the interpretation of results using wavelet-based approaches. The significance test establishes significance levels below which the results are considered not to be "significantly" different from zero and hence none of them can be used with sufficient confidence. The null hypothesis $\left(H_{0}\right)$ in the present work will be based on two independent Gaussian White Noise (GWN) time series and the associated sampling distributions of the interested wavelet-based quantities (statistics), which is a conventional approach in many statistical studies (e.g. Jenkins and Watts, 1969; TC98; Ge, 2007). In this approach, it is important to bear two points in mind. First, using the sampling distribution of the WCS (or WLC) of two independent GWNs is not the only way to establish the significance levels for the associated quantities. Two Gaussian red noise series (TC98), one time series of any kind (even without a known Fourier spectrum) and one GWN, or more particular combinations of two time series can all theoretically result in zero WCS and WLC and hence define significance levels of their own. For simplicity, we assume that the combination of two GWNs is not only the simplest but also the most representative kind for establishing the null hypothesis. The derived significance levels based on two GWNs can be readily extended for those based on two Gaussian red noise series (TC98). In the present work, therefore, only the case of two independent GWNs is considered in estimating the sampling distributions of the WCS and the WLC. The second point is that, in conventional approaches, the null hypothesis for a significance test for wavelet-based quantities is inevitably constructed on stationary reference (background) series such as GWNs and red noise series, even though the actual time series to be analysed often are nonstationary. Regarding this issue TC98 provided some reasons for still using stationary reference series. Their main point of view was that stationary significance tests are more mature and more suitable for providing a standard by which any nonstationarity can be detected. Moreover, we should point out here that, since the analysed nonstationary time series do not usually change their statistical properties dramatically over a short time, the use of stationary reference signals for establishing the null hypothesis is locally justified. Caution nevertheless should be taken when conducting significance testing on naturally observed data sets. 
The following discussions will be based on the Morlet wavelet, whose mother wavelet is defined as

$\psi(t)=\pi^{-1 / 4} e^{i \omega_{0} t} e^{-\frac{t^{2}}{2}}$

where $\omega_{0}$ is set equal to 6.0 and $t$ denotes time. The family of the Morlet wavelets $\psi_{a, b}(t)$ can be generated by time translations and scale dilations, such that

$\psi_{a, b}(t)=\frac{1}{\sqrt{a}} \psi\left(\frac{t-b}{a}\right)$.

The use of the Morlet wavelet and other complex wavelets will give rise to complex wavelet coefficients and hence additional information can be deduced from the phase angle of all complex wavelet quantities. The analyses in the following sections can readily be applied to other types of wavelets whenever the continuous wavelet transform is used. As for the significance tests on wavelet-based quantities obtained using the discrete wavelet transform, more considerations and manipulations are needed, which is beyond the scope of the present work.

\section{Significance test of the wavelet cross spectrum}

Two independent GWN series are denoted as $x$ and $y$ with variances $\sigma_{x}^{2}$ and $\sigma_{y}^{2}$, respectively. To avoid complication of notations, $x$ and $y$ also represent realisations of these two random processes.

The squared absolute value of the WCS of two GWNs is the product of two $\chi^{2}$-distributed random variables, $|X(a, b)|^{2}$ and $|Y(a, b)|^{2}$ (Eq. 2). TC98 gave a form of the probability density function (PDF) of the absolute value (not squared) of the WCS:

$$
f_{v}(z)=2^{2-v} z^{v-1} K_{0}(z) / \Gamma^{2}(v / 2)
$$

where $\Gamma$ is the Gamma function, $K_{0}(z)$ is the modified Bessel function of order zero, and $v$ is the degree of freedom of the $\chi^{2}$ distributions of the associated wavelet power. When complex wavelets are used, i.e. $v=2$, Eq. (7) becomes

$f_{\mathrm{TC}}(z)=z K_{0}(z)$

where the subscript "TC" indicates that it results from TC98's form Eq. (7). Equation (7), however, cannot be found in Jenkins and Watts (1968) as it seemed, nor was its derivation clearly presented. In what follows, we will provide a clear derivation of the sampling distribution of the WCS of two GWNs.

Wells et al. (1962) obtained the probability distribution of the product of two independent non-central $\chi^{2}$-distributed random variables, also reviewed later by Kotz and Srinivasan (1969). The sampling distribution of $\left|C_{x y}\right|^{2}$ based on two independent GWNs is a special case where the degrees of freedom of the two $\chi^{2}$ distributions are both 2 and the noncentrality parameters are both zero. More specifically, since

$\frac{|X|^{2}}{\delta t \sigma_{x}^{2} / 2} \sim \chi_{2}^{2}$

and

$\frac{|Y|^{2}}{\delta t \sigma_{y}^{2} / 2} \sim \chi_{2}^{2}$

with $\delta t$ being the sampling period or the reciprocal of the sampling frequency $F_{s}(\mathrm{Ge}, 2007)$, their product

$\frac{|X|^{2}}{\delta t \sigma_{x}^{2} / 2} \frac{|Y|^{2}}{\delta t \sigma_{y}^{2} / 2} \sim W_{2}$,

where $W_{2}$ denotes the probability distribution with a PDF

$f(z)=\frac{1}{2} K_{0}\left(z^{1 / 2}\right)$,

based on Wells et al. (1962). Rearranging terms we have

$\frac{\left|C_{x y}(a, b)\right|^{2}}{\sigma_{x}^{2} \sigma_{y}^{2}} \sim \frac{1}{4} \delta t^{2} W_{2}$.

The significance level for a percentile $\alpha$ can be deduced from the $1-\alpha$ percentile of the $W_{2}$ distribution. Moreover, we can prove that the results obtained here, Eq. (10), and TC98's form, Eq. (8), are equivalent to each other and are equally correct in determining the significance levels for the WCS of two GWNs. More details are given in Appendix A.

A set of simulated signals can be used to examine the proposed significance test of the WCS. Sine signals with a prescribed signal-to-noise ratio, SN, were generated. Same as those in Ge (2007), each of the signals consists of 2000 points with a sampling frequency of $50 \mathrm{~Hz}$ (i.e. $\delta t=0.02 \mathrm{~s}$ ). The entire period of 2000 points is divided into three intervals. In different intervals the two generated time series, $x(t)$ and $y(t)$, have different properties. The two signals are generated such that

$$
\left\{\begin{array}{l}
x=A \sin \left(2 \pi f_{x} t\right)+\mathrm{GWN}(0,1) \\
n \in[701,900] \\
y=A \sin \left(2 \pi f_{x} t+\frac{t-t(700)}{t(900)-t(700)} 2 \pi\right)+\mathrm{GWN}(0,1) \\
n \in[701,900] \\
x=A \sin \left(2 \pi f_{x} t\right)+\operatorname{GWN}(0,1) \\
n \in[901,1100] \\
y=A \sin \left(2 \pi f_{x} t-\frac{2}{3} \pi\right)+\mathrm{GWN}(0,1) \\
n \in[901,1100] \\
x=A \sin \left(2 \pi f_{x} t\right)+\mathrm{GWN}(0,1) \\
n \in[1101,1300] \\
y=A \sin \left(2 \pi f_{x} t+\frac{2}{3} \pi\right)+\mathrm{GWN}(0,1) \\
n \in[1101,1300]
\end{array}\right.
$$

where $n$ denotes the order of data points, $f_{x}$ is set at $8 \mathrm{~Hz}, A$ is determined by the prescribed signal-to-noise ratio, which is 3 for the present study, through the relation 


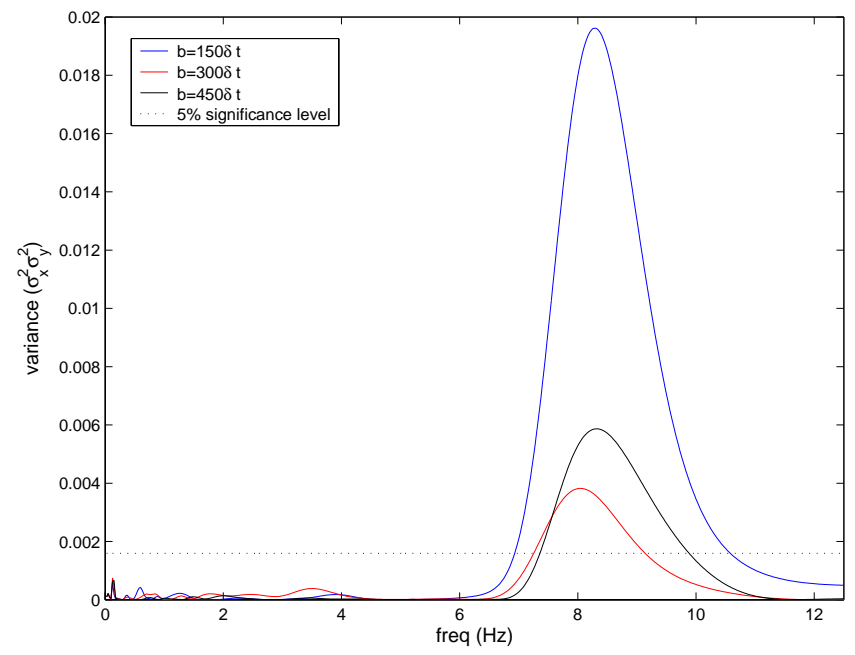

Fig. 1. Squared absolute value of the wavelet cross spectrum $\left|C_{x y}(a, b)\right|^{2}$ of two simulated signals given by Eq. (11) and the $5 \%$ significance level.

$\mathrm{SN}=10 \log _{10} A^{2}$, and $\operatorname{GWN}(0,1)$ denotes a GWN with a zero mean and a unit standard deviation. These two simulated signals thus have a linearly varying phase difference in the first interval $n \in[701,900]$, a constant phase difference $-2 \pi / 3$ in the second interval $n \in[901,1100]$, and a constant phase difference $2 \pi / 3$ in the third interval $n \in[1101,1300]$. The signals in the periods prior to $n=701$ and after $n=1300$ are just GWNs. TC98's Fortran code for estimating the wavelet coefficient was used with an adjustment as suggested in Appendix A of Ge (2007). To avoid the cone of influence (TC98), only the central 600 points $(n \in[701,1300])$ are used for analysis. Although the retained wavelet coefficients still are affected by the edge effect at the largest scales, the influence of the edges is negligible.

Figure 1 shows the squared absolute value of the WCS, $\left|C_{x y}\right|^{2}$, between the simulated signals $x$ and $y$ (Eq. 11) normalised by the product of the variances of the two signals over the period that covers the central 600 points. The three curves in Fig. 1 represent three vertical slices of $\left|C_{x y}\right|^{2}$ values in the original time-scale domain, with scale $a$ converted into frequency $f$ through the relation

$a=f_{c} / f \approx 0.9394 / f$

for the Morlet wavelet. The timing for the three curves, $b$, is expressed relative to the beginning of the central 600 points instead of that of the entire 2000 points. Hence, the times at $b=150 \delta t, b=300 \delta t$, and $b=450 \delta t$ fall in the three intervals in Eq. (11), respectively. With a relatively high $\mathrm{SN}$ ratio, all the three curves of $\left|C_{x y}\right|^{2}$ are well above the theoretical $5 \%$ significance level based on Eq. (10), regardless of the patterns of the phase difference between $x$ and $y$ in different intervals. As expected, all the three curves peak at about $8 \mathrm{~Hz}$ (i.e. $f_{x}$ ) with small deviations due to the added GWNs.
Very small ripples are observed in Fig. 1 at frequencies lower than $4 \mathrm{~Hz}$. These ripples are correctly indicated to be random noise, or not significantly different from zero with a $95 \%$ confidence, by the $5 \%$ significance level.

\section{Property of the phase angle of the WCS}

The phase angle of the WCS for each point in the twodimensional time-scale domain can be plotted (TC98). TC98, however, did not propose any significance test or confidence interval for the phase. The significance test on the phase angle appears not to be as important as that on other popular wavelet-based quantities. Nevertheless, some of the properties of the phase angle of the WCS might be interesting to the users of the wavelet analysis.

If the range of the phase angle $\phi$ is $[-\pi, \pi]$ and the conventional range of the arctangent function is $[-\pi / 2, \pi / 2]$, we have

$\phi=\arctan \frac{\operatorname{Im}\left[X^{*} Y\right]}{\operatorname{Re}\left[X^{*} Y\right]}+\mu \pi=\phi_{0}+\mu \pi$,

where $\mu=0$ when the complex number $C_{x y}=X^{*} Y$ is in the first or fourth quadrant of the complex plane, $\mu=1$ when $C_{x y}$ is in the second quadrant, and $\mu=-1$ when $C_{x y}$ is in the third quadrant. Using the real and imaginary parts of $X$ and $Y$, Eq. (13) leads to

$\tan \phi_{0}=\frac{\operatorname{Re}[X] \operatorname{Im}[Y]-\operatorname{Im}[X] \operatorname{Re}[Y]}{\operatorname{Re}[X] \operatorname{Re}[Y]+\operatorname{Im}[X] \operatorname{Im}[Y]}$.

When $x$ and $y$ are independent GWNs, the numerator and the denominator of Eq. (14) are independent of each other, unbounded, and can take any real values. As suggested by Jenkins and Watts (1968, p. 366), they can be considered to be approximately normally distributed variables. The quantity $\tan \phi_{0}$ is thus the ratio of two normally distributed random variables, which has a standard Cauchy distribution denoted as $C(0,1)$. More details are given in Appendix B. The $\mathrm{PDF}$ of $\tan \phi_{0}$ is therefore

$f(z)=\frac{1}{\pi\left(1+z^{2}\right)}$,

which yields no mean or any higher-order moments but a zero median. That Cauchy distributed random variables have no variance implies that the random variable can take extreme values more often than would naturally occur (e.g. for normally distributed variables). Given a sufficiently long set of time series $x$ and $y(\mathrm{GWNs}), \tan \phi_{0}$ can disperse into an unbounded range with extreme values observed more frequently than rarely. The angle $\phi_{0}$ can hence take any value between $-\pi / 2$ and $\pi / 2$ and is distributed much more uniformly than a normally distributed variable. Papoulis (1965, p. 199) reached a further result: $\phi_{0}$ in Eq. (14), as the principal part of the phase angle of a random complex number 
that has independently normally distributed real and imaginary parts with zero means and equal variances, has an exactly uniform distribution in the interval from $-\pi / 2$ to $\pi / 2$. Taking advantage of this observation as well as the fact that $C_{x y}=X^{*} Y$ can be in the four quadrants of the complex plane with equal probabilities, we deduce that the phase angle of $C_{x y}=X^{*} Y, \phi$, has a uniform distribution in the interval between $-\pi$ to $\pi$. Therefore, no significance level can be established with the aid of the sampling distribution of $\phi$. In other words, the phase angle $\phi$ will fill the range between $-\pi$ and $\pi$ without concentrating around any particular value. The randomness of the two GWNs $x$ and $y$ makes $\phi$ not likely to be bounded around any value but rather scattered in the whole range of $[-\pi, \pi]$.

Figure 2 shows the time variation of the phase angle $\phi$ of the WCS of the simulated signals $x$ and $y$ given by Eq. (11) at three frequencies: $6 \mathrm{~Hz}, 8 \mathrm{~Hz}$, and $10 \mathrm{~Hz}$. According to Eq. (11) the phase at $6 \mathrm{~Hz}$ and $10 \mathrm{~Hz}$ should reflect both randomness due to the added GWN components and the influence of the scale at $8 \mathrm{~Hz}$ through the correlation between adjacent scales. The phase pattern at $8 \mathrm{~Hz}$ is evident, including a linear increase in the first 200-point period, a relatively stable negative phase angle in the middle 200-point period, and a nearly constant positive phase angle in the last portion of the period. However, the nearly constant phase angles in the second and third intervals are not exactly $-2 \pi / 3$ and $2 \pi / 3$ as prescribed by Eq. (11). This is not completely unexpected since the phase difference between $x$ and $y$ (Eq. 3) at any frequency has been perturbed by the different GWN components added to $x$ and $y$. Affected by the scale at $8 \mathrm{~Hz}$, the phase angle of the WCS at $10 \mathrm{~Hz}$ bears resemblance to that at $8 \mathrm{~Hz}$, while the pattern of the phase appears to be much more irregular. The phase angle at $6 \mathrm{~Hz}$ reflects randomness with values close to $\pm \pi$ occurring more often than rarely, especially within the interval of the last 200 points. The difference between the phase patterns at $8 \mathrm{~Hz}$ and those at $6 \mathrm{~Hz}$ and $10 \mathrm{~Hz}$ are consistent with the deduced properties of the phase angle of the WCS of two GWNs. It hence appears that if the phase difference between two signals has a regular pattern the phase of the WCS tends to become stable and reveal that pattern rather than fluctuate everywhere. The significance test of the phase angle of the WCS should thus be replaced by identification of regular phase patterns. Since it is still quite subjective to distinguish a regular phase pattern from randomly fluctuating ones, we suggest that the wavelet linear coherence of the same two signals be inspected. More details are given in Sect. 4.

\section{Significance test of the wavelet linear coherence}

The definition of the WLC is given by Eq. (4). For a certain $a$, the discrete form of Eq. (4) can be expressed as

$L_{x y}(a)=\frac{\left|\sum_{m} X_{a}^{*}(b) Y_{a}(b)\right|^{2}}{\sum_{m}\left|X_{a}(b)\right|^{2} \sum_{m}\left|Y_{a}(b)\right|^{2}}$,
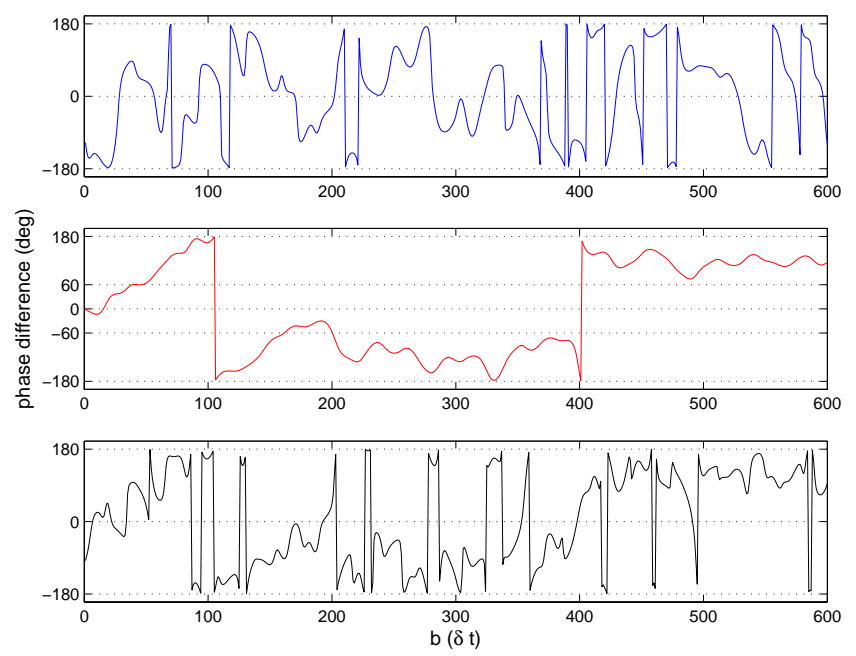

Fig. 2. Phase angle of the wavelet cross spectrum, $\phi(a, b)$ $(\phi \in[-\pi, \pi])$, of two simulated signals given by Eq. (11) at $6 \mathrm{~Hz}$ (blue), $8 \mathrm{~Hz}$ (red), and $10 \mathrm{~Hz}$ (black).

where the summations are over $m$ data points such that $T=m \delta t$. This form of the WLC can be readily viewed as a squared correlation coefficient between two $m$-point time series: $\left\{X_{a}^{*}\right\}$ and $\left\{Y_{a}\right\}$. As Jenkins and Watts (1968, p. 379) pointed out, the sampling distribution of the correlation coefficient (not squared) of two normally distributed random variables can be obtained by Fisher's z-transformation. Then the only problem to consider now is how the two series, $\left\{X_{a}^{*}\right\}$ and $\left\{Y_{a}\right\}$, can be treated as realisations of two random variables.

The continuous wavelet transform as used in the present work gives rise to redundancy when one-dimensional time series is resolved into a two-dimensional time-scale domain. Neither of the two series, $\left\{X_{a}^{*}(j)\right\}$ and $\left\{Y_{a}(j)\right\}$ $(j=1,2, \cdots, m)$, can be considered to be a series of independent observations unless the inter-correlation of adjacent data points in $\left\{X_{a}^{*}\right\}$ and $\left\{Y_{a}\right\}$ is removed. It can be deduced that the covariance of two wavelet coefficients of a GWN series $x$ at scale $a$ and times $b_{i}$ and $b_{j}$, i.e.

$\left|\operatorname{Cov}\left[X_{a}\left(b_{i}\right), X_{a}\left(b_{j}\right)\right]\right|=\delta t \sigma_{x}^{2} e^{-\Delta b^{2} / 4 a^{2}}$,

where $\Delta b=\left|b_{i}-b_{j}\right|$ (Appendix C). The decay of the correlation coefficient of wavelet coefficients of the same scale with a separation in time $\Delta b$ is determined by the exponential function $e^{-\Delta b^{2} / 4 a^{2}}$, or

$\rho(\Delta b)=e^{-\Delta b^{2} / 4 a^{2}}$

with $\rho(\Delta b)$ denoting the absolute value of the correlation coefficient of temporally adjacent wavelet coefficients. Obviously, farther apart wavelet coefficients are less correlated. More discussion can be found in Appendix C.

At this point we can prescribe a decorrelation parameter $\kappa$ such that the correlation coefficient $\rho(\Delta b)=1 / \kappa$. Solving 


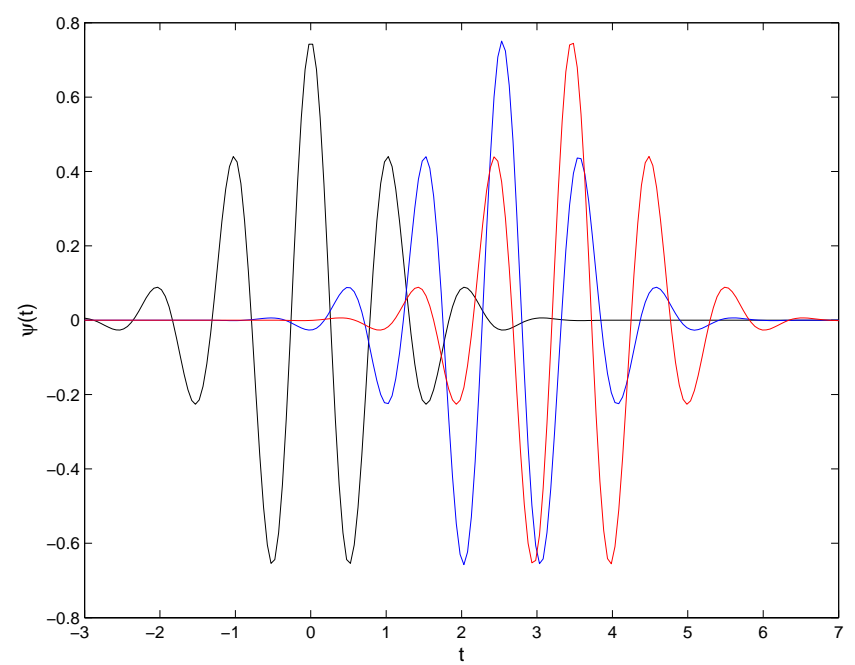

Fig. 3. Decorrelation of wavelet coefficients of a GWN with an increasing time separation reflected by the decorrelation of wavelets. The mother Morlet wavelets $\psi(t)$ are used for illustration. The blue and the black wavelets have a correlation coefficient of $1 / 5(\kappa=5)$; the red and the black wavelets have a correlation coefficient of $1 / 20$ $(\kappa=20)$.

for $\Delta b$ we are able to determine the time separation $\Delta b$ beyond which the correlation coefficient between wavelet coefficients falls below $1 / \kappa$. The decorrelation parameter $\kappa$ can be set at a large positive number such as 5 or 20 , corresponding to correlation levels of $1 / 5$ or $1 / 20$, respectively. We can further assume that the wavelet coefficients are considered to be independent of each other when their correlation coefficient is below $1 / \kappa$. In this approach, a series of wavelet coefficients, such as $\left\{X_{a}^{*}(j)\right\}(j=1,2, \cdots, m)$, can be grouped into a number of independent sub-series. Within any individual sub-series the correlation coefficient of any two wavelet coefficients is larger than $1 / \kappa$, while the wavelet coefficients belonging to different sub-series have a correlation coefficient lower than $1 / \kappa$. Therefore, each sub-series is equivalent to an independent observation in a sample, and the number of the sub-series is equivalent to the sample size. More specifically, setting $\rho(\Delta b)=1 / \kappa$ yields

$\Delta b=2 \sqrt{\ln \kappa} a$.

The sample size, denoted as $N(a)$ which is a function of the scale, can be estimated through the relation $N(a)=T / \Delta b$, so that

$N(a)=\frac{m \delta t}{2 \sqrt{\ln \kappa} a}$.

Since the correlation coefficient of the wavelet coefficients of a GWN $y$ decays at the same rate as described by Eq. (18), Eq. (20) is also applicable to the wavelet coefficient of $y$. For example, for the case of $a=1$ (the mother Morlet wavelet), the time separation $\Delta b$ for $\kappa=5$ is $2 \sqrt{\ln 5}$ and that for $\kappa=20$ is $2 \sqrt{\ln 20}$. The Morlet wavelets with these two separations from a Morlet wavelet at the origin are shown in Fig. 3, giving an idea about how wavelet coefficients become decorrelated as they are more and more separated in time. The correlation coefficient of wavelet coefficients essentially is reflected by that of wavelet functions when the analysed time series is a GWN (Appendix C). For clarity, the Morlet wavelets (real part), instead of the wavelet coefficients, are shown in Fig. 3. Figure 3 also indicates that increasing $\kappa$ from 5 to 20 does not require a much wider separation. Hence, we will simply set $\kappa$ at 5 for most cases in the following sections.

The two original series $\left\{X_{a}^{*}\right\}$ and $\left\{Y_{a}\right\}$ of length $m$ can now be treated as two reduced series of independent observations of length $N(a)$. Using Fisher's transformation we have

$$
\arctan h\left(r_{x y}\right) \sim N\left(\arctan h\left(\rho_{x y}\right), \frac{1}{\sqrt{N(a)-3}}\right),
$$

where $r_{x y}$ and $\rho_{x y}$ are the theoretical and estimated correlation coefficients of $\left\{X_{a}^{*}\right\}$ and $\left\{Y_{a}\right\}$. In the case of $x$ and $y$ being GWNs and a large $N(a)$ (i.e. at reasonably small scales), the probability distribution is approximately

$$
\arctan h\left(r_{x y}\right) \sim N\left(0, \frac{1}{\sqrt{N(a)}}\right) \approx N\left(0, \sqrt{\frac{2 \sqrt{\ln \kappa} a}{m \delta t}}\right) .
$$

The $\alpha$ significance level of the WLC is hence

$$
D_{\alpha}\left(L_{x y}\right)=\tanh ^{2}\left(s_{\alpha} \sqrt{\frac{2 \sqrt{\ln \kappa} f_{c} F_{s}}{m f}}\right)
$$

with $s_{\alpha}$ denoting the $1-\alpha$ percentile of the standard normal distribution and scales converted into frequencies through Eq. (12). More detailed derivation can be found in Appendix $\mathrm{C}$. When $N(a)$ is close to or even smaller than 3 , as is often the case at very large scales, the resulting $\alpha$ significance level according to Eqs. (21) and (22) tends to be larger than one. For most cases, therefore, no WLC results should be trusted with confidence at the largest scales.

Van Milligen et al. (1995) estimated the "statistical noise level" of the WLC as

$D_{V M}\left(L_{x y}\right) \approx 2 \sqrt{\frac{F_{s}}{m f}}$

through a similar but much more heuristic reasoning. We believe that their so-called statistical noise level is exactly the significance level, but it is not clear what $\alpha$ value was associated with Eq. (24). Comparing Eqs. (23) and (24) we have an impression that Eq. (23), with more factors taken into account, is a much more accurate form than the one suggested by van Milligen et al. (1995).

As an example, Fig. 4 shows two sets of significance levels. The blue set of curves is significance levels for $m$ of 40 , $80,140,200$, and 400 points, from top to bottom, for the case 


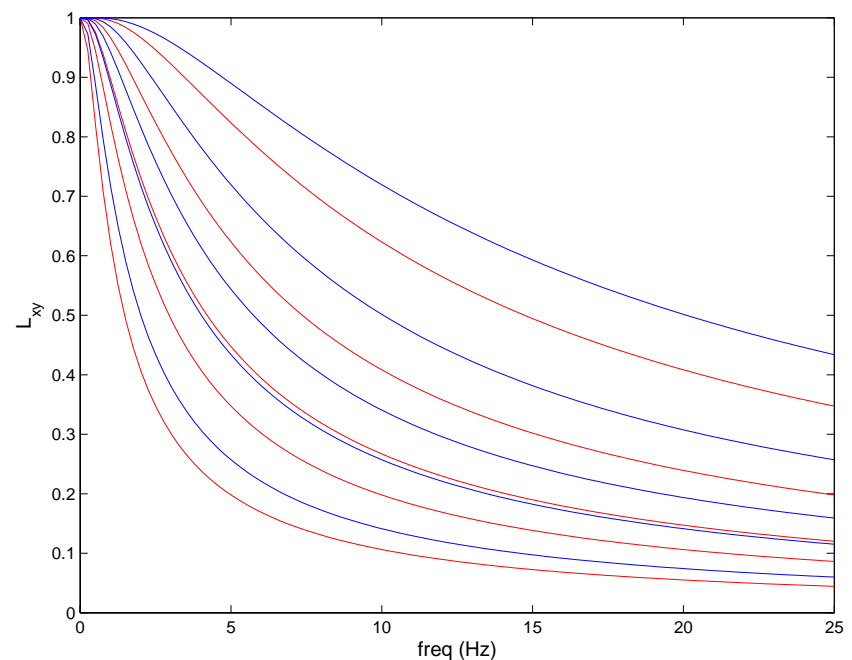

Fig. 4. 5\% significance levels of the wavelet linear coherence: red: $\kappa=5, m=40,80,140,200$, and 400 from top to bottom; blue: $\kappa=20$, $m=40,80,140,200$, and 400 from top to bottom.

of $\kappa=20$. It is obvious that the low frequency range tends to be seriously affected by random noise, and the significance levels drop rapidly with increasing $m$. When the decorrelation condition is relaxed to $\kappa=5$, the resulting significance levels (the red set of curves) are evidently, but not considerably, lower than their corresponding ones for $\kappa=20$. For example, for $m=80$, the difference of the significance levels between $\kappa=20$ and $\kappa=5$ is approximately 0.08 for most of the frequencies. The difference seems to be much smaller when $m$ is increased to 200 or 400 . In most practical cases, therefore, $\kappa=5$ should be reasonably effective. Another feature of Fig. 4 is that all significance level curves are below one. This is not realistic because Eq. (23) is only applicable to scales that are not too large. One should be aware of the possibility of $N(a)$ being close to 3 , and, practically, should not make use of any results below $5 \mathrm{~Hz}$ in Fig. 4 .

Figure 5 shows three WLC curves starting at $b=50 \delta t$, $250 \delta t$, and $450 \delta t$ with an integration length $m$ of 120 points $(\kappa=5)$, so that the three WLC curves are completely estimated within the three intervals respectively. In Fig. 5, the WLC levels around $8 \mathrm{~Hz}$ for the second and third intervals are well above the local 5\% significance level, while that for the first interval cannot be distinguished from the WLC caused by random noise. This clearly exhibits the effect of the phase-difference pattern of $x$ and $y$ on the resulting WLC values. During the second and third intervals, the stability in the phase difference (Fig. 2) yields a peak in the WLC at the associated frequency. In contrast, the linearly varying, i.e. inconstant, phase difference between $x$ and $y$ in the first interval leads to a low level of WLC that is considered not to be significantly larger than zero. Furthermore, the rapid fluctuations at low frequencies, which have obviously resulted from randomness, are collectively far below the $5 \%$ significance

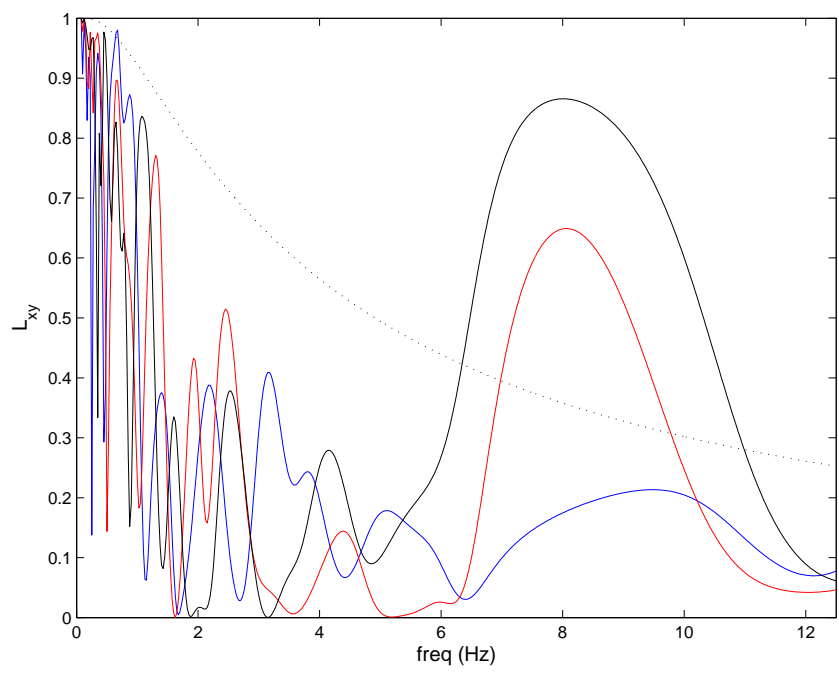

Fig. 5. Wavelet linear coherence $L_{x y}$ of two simulated signals given by Eq. (11) estimated over $m=120$ points starting from $b=50 \delta t$ (blue), $b=250 \delta t$ (red), and $b=450 \delta t$ (black). The three WLC curves represent, respectively, the linear relations in the three intervals as in Eq. (11).

level, especially from $1 \mathrm{~Hz}$ to $5 \mathrm{~Hz}$. This implies the possibility of relaxing $\alpha$ to larger values, such as $10 \%$ or $20 \%$, for this particular case.

\section{Significance tests on actually observed data sets}

As pointed out previously, theoretically developed significance tests should be examined and validated by actually observed data, simply because assumptions, simplifications, and approximations have been inevitably made in the course of derivation. The NOAA's (National Oceanic and Atmospheric Administration) Lake Michigan wind-wave data set is used here again. The description of the data set can be found in Ge and Liu (2007 and 2008) and Ge (2007). Briefly, the horizontal wind velocity time series (denoted as $u$ ) and the wave elevation (denoted as $\eta$ ) were simultaneously recorded by a NOAA 3-m discus buoy, deployed during the autumn of 1997 in nearshore eastern Lake Michigan of the United States $\left(43.02^{\circ} \mathrm{N}, 86.27^{\circ} \mathrm{W}\right)$ with a sampling frequency $F_{s}=1.7 \mathrm{~Hz}$. The data set exhibits a long process of wave growth in response to increasing wind forcing, as discussed previously by Ge and Liu (2008).

A 2000-point data segment was picked out from the windwave data set, and the central 600-point wavelet coefficients were used for further analysis. The WLC $L_{\eta u}$ estimated over the period from $b=200 \delta t$ to $299 \delta t(m=100$ and $T=58.8 \mathrm{~s})$ is shown in Fig. 6 against the 5\% and 20\% significance levels. Two peaks in the WLC are well above the 5\% significance level, one at $0.15 \mathrm{~Hz}$ and the other around $0.5 \mathrm{~Hz}$. The broader peak around $0.5 \mathrm{~Hz}$ is in fact a frequency range from 


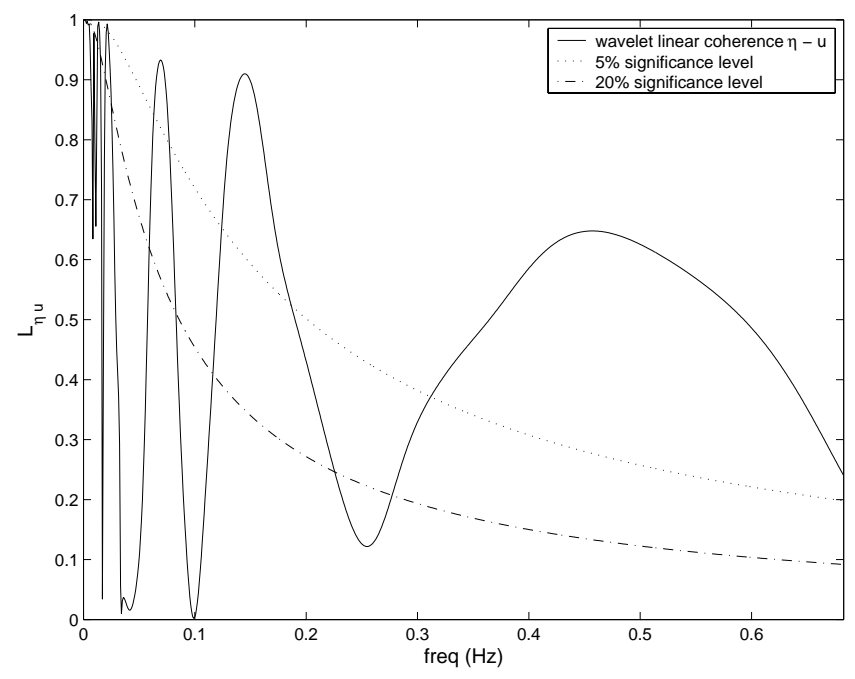

Fig. 6. Wavelet linear coherence of the wave elevation $\eta$ and the wind velocity fluctuations $u$ estimated over the period from $b=200 \delta t$ to $b=299 \delta t(m=100)$, compared with the $5 \%$ and $20 \%$ significance levels.

$0.35 \mathrm{~Hz}$ to $0.65 \mathrm{~Hz}$ in which all frequency components in the winds are linearly coupled with those in the waves, because the width of this peak should not be the consequence of locally poor resolution in frequency. A third peak at $0.07 \mathrm{~Hz}$ is also above the 5\% significance level with a high WLC value of 0.94 . Since the local significance level is almost equally high (about 0.84), we do not consider this peak to be statistically significant.

The phase difference between the wave and wind time series, or the phase angle of their WCS $C_{\eta u}$ at three representative frequencies, $0.15 \mathrm{~Hz}$ (for extremely high WLC), $0.28 \mathrm{~Hz}$ (for extremely low WLC), and $0.5 \mathrm{~Hz}$ (for locally high WLC), is shown in Fig. 7. From $b=200 \delta t$ to $299 \delta t$, the phase difference at $0.15 \mathrm{~Hz}$ is very stable around $\pm 180^{\circ}$ (note that the large steps are artificially caused by the definition of the phase angle), indicating that the wind and wave time series are almost perfectly out of phase at $0.15 \mathrm{~Hz}$ during the time period from $b=200 \delta t$ to $299 \delta t$. This characteristic is however not as evident in other time periods. At $0.5 \mathrm{~Hz}$ in the same period, the phase difference also appears to be centered around $180^{\circ}$, while its deviation from $180^{\circ}$ is relatively larger than that at $0.15 \mathrm{~Hz}$ (e.g. from $b=230 \delta t$ to $260 \delta t$ ). In comparison, the phase difference in the same period at $0.28 \mathrm{~Hz}$ exhibits a much more random pattern. The phase angle does not seem to settle around any particular value. The different characteristics of the phase angle at these three frequencies in the studied 100-point period are consistent with the deduced properties of the phase angle of the WCS and its relation to the WLC in the previous sections. Consequently, a significance test of the WLC can be used to help identify meaningful patterns in the phase angle of the WCS.
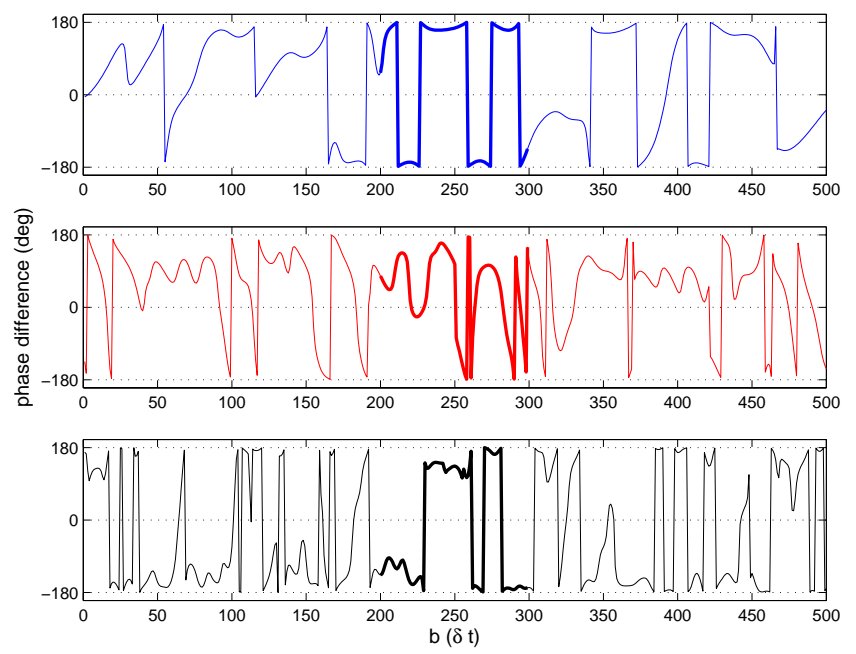

Fig. 7. Phase angle of the wavelet cross spectrum $C_{\eta u}$ at $0.15 \mathrm{~Hz}$ (blue), $0.28 \mathrm{~Hz}$ (red), and $0.5 \mathrm{~Hz}$ (black). Wide curves of all colors highlight the period from $b=200 \delta t$ to $b=299 \delta t$.

Figure 7 also reveals the randomness and intermittency of the phase angle. During the period from $b=50 \delta t$ to $150 \delta t$, for example, the wind velocity fluctuations lead the wave elevation by a linearly varying phase angle growing from $-180^{\circ}$ to $180^{\circ}$ from $50 \delta t$ to $100 \delta t$ and then growing from 0 to $180^{\circ}$ from $110 \delta t$ to $150 \delta t$ at $0.15 \mathrm{~Hz}$. At $0.28 \mathrm{~Hz}$ and in the same interval, however, the winds appear to lead the waves by a relatively constant phase angle of approximately $90^{\circ}$, which is only briefly interrupted by a sharp peak at around $b=100 \delta t$. According to Sect. 4 , this pattern implies a relatively high WLC value over that period. At $0.5 \mathrm{~Hz}$, the winds lag behind the waves by about $160^{\circ}$ in most part of the same period, while from $b=100 \delta t$ to $120 \delta t$ the phase difference between the winds and the waves is slightly larger than $180^{\circ}$, so that the angle wraps around and appears to be $+180^{\circ}$.

\section{Conclusion}

Significance testing is an important step in interpreting results based on the wavelet analysis and wavelet-based spectral moments. In many cases, in practice, even large peaks could be merely artifacts resulting from randomness due to the nature of the problem and errors in the measurements. Significance testing helps to establish a basis upon which one can reject a peak value in the wavelet-based quantity or accept it with a degree of confidence. It becomes even more important when the wavelet analysis, initially developed as an exploratory tool, is being merged into the system of statistics. In the scenario of statistical signal processing, observed data records should be viewed as realisations of stationary or nonstationary random processes, and all large values in the results are not considered to be equally meaningful. 
Interpretation of results using wavelet-based techniques then should be done in the same manner as for statistical inference.

On the other hand, significance testing on wavelet-based quantities (now treated as wavelet-based "statistics") can be extremely difficult, because the wavelet analysis is most frequently used for processing nonstationary time series. When using sampling distributions based on background noise time series to establish the null hypothesis, we are clear that the choice of the background noise signals can be subjective and arbitrary. Instead of examining an exhaustive list of background noise series, the present work is focused on a representative combination, two independent Gaussian white noise series. Sampling distributions of the WCS and the WLC of two independent GWN series are estimated through rigorous statistical reasoning. When other combinations of reference series are preferred, the same procedure can be followed to obtain their associated sampling distributions. Significance levels of these two wavelet-based quantities are then deduced based on the sampling distributions.

In the present work, the sampling distributions of the WCS and the WLC are derived by analytical approaches, which are preferred by TC98. In many cases where the analytical approach seems to be difficult, Monte Carlo simulations can be employed (TC98). The Monte Carlo simulation, however, has disadvantages of high computation cost and the inability to provide the user with a clear mathematical form for the sampling distributions or the significance levels. Through simulated noisy sinusoidal signals and actual wind-wave time series observed from Lake Michigan, it is shown that the analytically developed significance tests are reasonably effective in distinguishing large WCS and WLC values that indicate meaningful linear spectral relations from those generated by random noise. Furthermore, in the absence of significance tests for the wavelet phase difference between time series (i.e. the phase angle of the WCS), the significance test of the WLC can help identify meaningful patterns in the phase difference. In contrast to earlier applications of the wavelet analysis (e.g. Foufoula-Georgiou and Kumar, 1994), significance tests help to place the interpretation of results on a rigorous statistical basis, so that the interpretation no longer depends on the researcher's personal experience and judgment.

Significance tests of third-order wavelet spectral moments, such as the wavelet bispectrum and the wavelet bicoherence (van Milligen, 1995; Ge and Liu, 2007; Elsayed, 2008), will be investigated in a future work.

\section{Appendix A}

\section{Sampling distribution of the WCS given by TC98}

According to Eq. (10), the $\alpha$ significance level of $\left|C_{x y}(a, b)\right|^{2} / \sigma_{x}^{2} \sigma_{y}^{2}$ is $\delta t^{2} Z(1-\alpha) / 4$, where $Z(1-\alpha)$ is the $1-\alpha$ percentile of the $W_{2}$ distribution. Alternatively, since the conventionally defined wavelet coefficient of $x(t)$ at time $b$ and scale $a$, i.e. $X(a, b)$, and the wavelet coefficient of $x(t)$ at time $t_{n}$ and scale $a$ defined by TC98, i.e. $W_{n}^{x}(a)$, are related by $|X(a, b)|^{2}=\delta t\left|W_{n}^{x}(a)\right|^{2}(\mathrm{Ge}, 2007)(\mathrm{a}$ similar relation holds for $y(t))$, the $\alpha$ significance level of $\left|W_{n}^{x}(a) W_{n}^{y}(a)\right|^{2} / \sigma_{x}^{2} \sigma_{y}^{2}$ becomes $Z(1-\alpha) / 4$. Therefore the $\alpha$ significance level of $\left|W_{n}^{x}(a) W_{n}^{y}(a)\right| / \sigma_{x} \sigma_{y}$ is $\sqrt{Z(1-\alpha)} / 2$. Compared with TC98's result that the $\alpha$ significance level of $\left|W_{n}^{x}(a) W_{n}^{y}(a)\right| / \sigma_{x} \sigma_{y}$ is $Z_{T C}(1-\alpha) / 2$ for GWNs $x$ and $y$ and complex wavelets $(\nu=2)$ with $Z_{T C}(1-\alpha)$ denoting the $1-\alpha$ percentile of the distribution given by Eq. (8), the only question now remaining is whether $Z_{T C}(1-\alpha)$ is equal to $\sqrt{Z(1-\alpha)}$. We only have to consider two PDFs: Eq. (8) given by TC98 without any explanation and Eq. (9) as a particular case of the results given by Wells et al. (1962).

The cumulant probability functions (CDF) can be obtained from Eqs. (8) and (9), respectively. The CDF based on TC98's form is

$F_{T C}(z)=1-z K_{1}(z)$

and the CDF associated with the PDF given by Eq. (9) is

$F(z)=1-\sqrt{z} K_{1}(\sqrt{z})$

for any real positive $z$. It is obvious immediately that $Z_{T C}(1-\alpha)=\sqrt{Z(1-\alpha)}$ holds for any $\alpha$ value from zero to one. TC98's forms, Eqs. (7) and (8), are hence reconciled with Eq. (9) developed in the present work.

\section{Appendix B}

\section{More details on the sampling distribution of the phase angle of the WCS}

A property will be useful for the following discussion: if two time series (more rigorously, random variables or stationary random processes) are independent, the mean value of their product is the product of their respective means, i.e.

$$
\begin{aligned}
E[x y] & =\iint x y f(x, y) d x d y \\
& =\iint x y f(x) f(y) d x d y \\
& =E[x] E[y],
\end{aligned}
$$

where $E[\cdots]$ means the expectation, $f(x, y)$ denotes the joint probability density function of $x$ and $y$, and $f(x)$ and $f(y)$ are the marginal probability density functions of $x$ and $y$, respectively.

From Eq. (14), $\tan \phi_{0}$ is the ratio of $A=\operatorname{Re}[X] \operatorname{Im}[Y]-\operatorname{Im}[X] \operatorname{Re}[Y]$ to $B=\operatorname{Re}[X] \operatorname{Re}[Y]+\operatorname{Im}[X] \operatorname{Im}[Y]$. It is easy to see that they can be both positive and negative with unbounded values. Jenkins and Watts (1968, p. 366) suggested that 


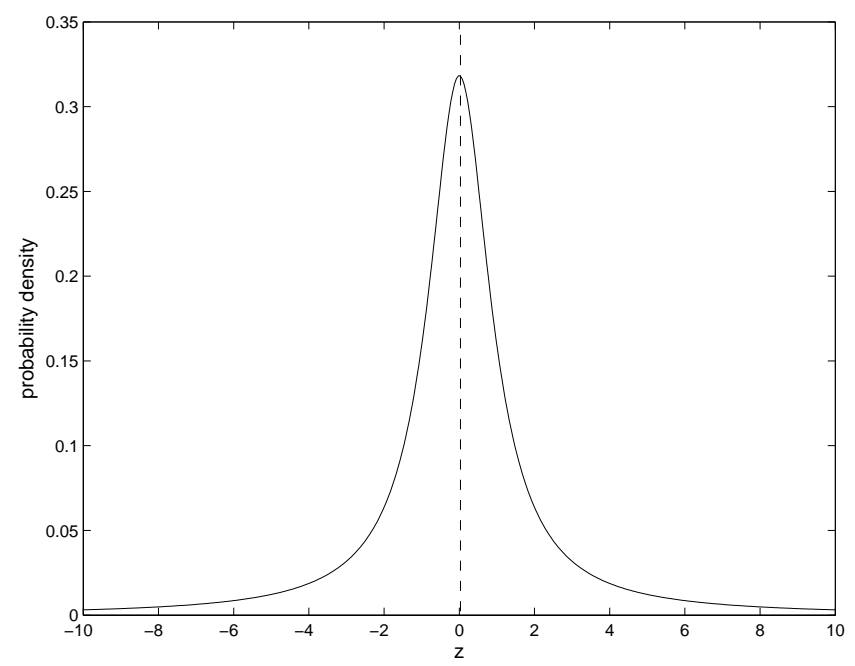

Fig. B1. Probability density function of the standard Cauchy distribution $C(0,1)$.

they can be approximately treated as normally distributed variables. Following the same approach that leads to the independency of the real and imaginary parts of the wavelet coefficient of a GWN (Ge, 2007), we have the independency between any two of $\operatorname{Re}[X], \operatorname{Im}[X], \operatorname{Re}[Y]$, and $\operatorname{Im}[Y]$. Therefore, also using Eq. (B1),

$$
\begin{aligned}
E[A] & =E[\operatorname{Re}[X] \operatorname{Im}[Y]]-E[\operatorname{Im}[X] \operatorname{Re}[Y]] \\
& =E[\operatorname{Re}[X]] E[\operatorname{Im}[Y]]-E[\operatorname{Im}[X]] E[\operatorname{Re}[Y]]=0,
\end{aligned}
$$

and similarly $E[B]=0$. Hence,

$$
\begin{aligned}
\operatorname{Var}[A]= & E\left[A^{2}\right] \\
= & E\left[\operatorname{Re}^{2}[X] \operatorname{Im}^{2}[Y]+\operatorname{Im}^{2}[X] \operatorname{Re}^{2}[Y]\right. \\
& -2 \operatorname{Re}[X] \operatorname{Im}[Y] \operatorname{Im}[X] \operatorname{Re}[Y]] \\
= & E\left[\operatorname{Re}^{2}[X]\right] E\left[\operatorname{Im}^{2}[Y]\right]+E\left[\operatorname{Im}^{2}[X]\right] E\left[\operatorname{Re}^{2}[Y]\right] .
\end{aligned}
$$

Since $\operatorname{Re}[X], \operatorname{Im}[X], \operatorname{Re}[Y]$, and $\operatorname{Im}[Y]$ of GWN $x$ and $y$ all have zero means,

$\operatorname{Var}[A]=\operatorname{Var}[\operatorname{Re}[X]] \operatorname{Var}[\operatorname{Im}[Y]]+\operatorname{Var}[\operatorname{Im}[X]] \operatorname{Var}[\operatorname{Re}[Y]]$, which yields

$\operatorname{Var}[A]=\frac{1}{2} \delta t^{2} \sigma_{x}^{2} \sigma_{y}^{2}$

Similarly, $B$ has the same variance.

With zero means, their covariance

$$
\begin{aligned}
\operatorname{Cov}[A, B]= & E[A B] \\
= & E[(\operatorname{Re}[X] \operatorname{Im}[Y] \\
& -\operatorname{Im}[X] \operatorname{Re}[Y])(\operatorname{Re}[X] \operatorname{Re}[Y] \\
& +\operatorname{Im}[X] \operatorname{Im}[Y])] .
\end{aligned}
$$

Expanding the right side of the equality above and using the properties of $\operatorname{Re}[X], \operatorname{Im}[X], \operatorname{Re}[Y]$, and $\operatorname{Im}[Y]$, we have

$\operatorname{Cov}[A, B]=0$.
Therefore, the two random variables, $A$ and $B$, both have an approximately normal distribution $N\left(0, \delta t \sigma_{x} \sigma_{y} / \sqrt{2}\right)$ and are independent of each other. Their ratio, $\tan \phi_{0}$, has a standard Cauchy distribution $C(0,1)$, whose PDF is expressed as

$f(z)=\frac{1}{\pi\left(1+z^{2}\right)}$

and is shown in Fig. B1. The PDF of the Cauchy distribution in Fig. B1 has a sharp peak at the origin and quite heavy tails at positive and negative ends. Because of the slow decay of the PDF values at large positive and negative $z$, integrals such as $\int_{-\infty}^{\infty} z^{n} f(z) d z$ do not exist in the real domain. The Cauchy distribution hence has no moments of any order, not even a mean value. Zero is obviously its median, but a median is not meaningful in determining its significance levels or confidence intervals. One might also attempt to estimate the integrals, such as $\int_{-z_{0}}^{z_{0}} f(z) d z$ and $\int_{z_{0}}^{\infty} f(z) d z$, to determine the critical value $z_{0}$ for establishing an associated significance level. In fact, however, neither of them converges in the real domain. For example, we have

$\int_{z_{0}}^{\infty} \frac{d z}{\pi\left(1+z^{2}\right)}=\frac{1}{2 \pi}\left[\pi+i\left(\ln \left(1+z_{0} i\right)-\ln \left(1-z_{0} i\right)\right)\right]$,

which is not a real number unless $z_{0}=0$, a trivial case.

\section{Appendix C}

\section{More details on the sampling distribution of the WLC}

First of all, more detailed derivation of Eq. (17) is given. Denoting the wavelets evaluated at $\left(a, b_{i}\right)$ and $\left(a, b_{j}\right)$ as $\psi_{a, i}$ and $\psi_{a, j}$ respectively, we have

$$
\begin{aligned}
\operatorname{Cov} & {\left[X_{a}\left(b_{i}\right), X_{a}\left(b_{j}\right)\right] } \\
& =\operatorname{Cov}\left[\int x(t) \psi_{a, i}^{*}(t) d t, \int x(t) \psi_{a, j}^{*}(t) d t\right] \\
& =E\left[\int x(t) \psi_{a, i}^{*}(t) d t \int x(t) \psi_{a, j}^{*}(t) d t\right]
\end{aligned}
$$

due to the zero-mean property. Therefore,

$$
\begin{aligned}
\operatorname{Cov} & {\left[X_{a}\left(b_{i}\right), X_{a}\left(b_{j}\right)\right] } \\
& =E\left[\iint x(t) x\left(t^{\prime}\right) \psi_{a, i}^{*}(t) \psi_{a, j}^{*}\left(t^{\prime}\right) d t d t^{\prime}\right] \\
& =\iint E\left[x(t) x\left(t^{\prime}\right)\right] \psi_{a, i}^{*}(t) \psi_{a, j}^{*}\left(t^{\prime}\right) d t d t^{\prime} \\
& =\delta t \sigma_{x}^{2} \int \psi_{a, i}^{*}(t) \psi_{a, j}^{*}(t) d t .
\end{aligned}
$$

Taking the absolute value of both sides of the equation above,

$$
\left|\operatorname{Cov}\left[X_{a}\left(b_{i}\right), X_{a}\left(b_{j}\right)\right]\right|=\delta t \sigma_{x}^{2}\left|\int \psi_{a, i}^{*}(t) \psi_{a, j}^{*}(t) d t\right| .
$$


In Ge (2007) it was defined that $I_{2}=\left|\int \psi_{a, i}(t) \psi_{a, j}(t) d t\right|^{2}$, and hence

$$
\left|\operatorname{Cov}\left[X_{a}\left(b_{i}\right), X_{a}\left(b_{j}\right)\right]\right|=\delta t \sigma_{x}^{2} \sqrt{I_{2}} .
$$

Using the results in $\mathrm{Ge}(2007)$ that

$$
I_{2}=e^{-\Delta b^{2} / 2 a^{2}}
$$

with $\Delta b=\left|b_{i}-b_{j}\right|$, we reach Eq. (17).

Moreover, since

$$
\begin{aligned}
\operatorname{Var}[X(a, b)] & =\operatorname{Var}[\operatorname{Re}[X]+i \operatorname{Im}[X]] \\
& =\operatorname{Var}[\operatorname{Re}[X]]+\operatorname{Var}[\operatorname{Im}[X]],
\end{aligned}
$$

using results in $\mathrm{Ge}$ (2007) we obtain

$$
\operatorname{Var}[X(a, b)]=\delta t \sigma_{x}^{2} .
$$

Comparing Eqs. (17) and (C3), we immediately have

$$
\begin{aligned}
\rho(\Delta b) & =e^{-\Delta b^{2} / 4 a^{2}} \\
& =\left|\int \psi_{a, i}^{*}(t) \psi_{a, j}^{*}(t) d t\right|,
\end{aligned}
$$

i.e. Eq. (18), where $\rho$ is the correlation coefficient of the wavelet coefficients that are separated by $\Delta b$. Particularly, when $\Delta b=0, \rho(0)=1$.

Furthermore, Eq. (C1) indicates that the covariance of two wavelet coefficients of a GWN that are separated in time is determined by the integral $\left|\int \psi_{a, i}^{*}(t) \psi_{a, j}^{*}(t) d t\right|$. This integral can be viewed as the covariance of two wavelets, one at $\left(a, b_{i}\right)$ and the other at $\left(a, b_{j}\right)$. Noting that when $\Delta b=0$ the integral becomes

$$
\left|\int \psi_{a, i}^{*}(t) \psi_{a, i}^{*}(t) d t\right|=\left.\sqrt{I_{2}}\right|_{\Delta b=0}=1
$$

this kind of covariance has the same properties as the absolute value of a correlation coefficient. Consequently, the correlation coefficient between wavelet coefficients, $\rho(\Delta b)$, can be expressed as the correlation coefficient between two wavelets that are at the same locations as their associated wavelet coefficients in the time-scale domain. This justifies the use of wavelets in Fig. 3 to show the decorrelation of wavelet coefficients with an increasing time separation.

Acknowledgements. The author wishes to thank the Ecosystems Research Division of the USEPA (NERL) and the Research Associateship Programs of the National Research Council for their resources and financial support.

Topical Editor S. Gulev thanks C. Torrence and another anonymous referee for their help in evaluating this paper.

\section{References}

Elsayed, M. A. K.: Nonlinear wave-wave interactions, J. Coastal Res., 24, 798-803, 2008.

Foufoula-Georgiou, E. and Kumar, P. (Eds.): Wavelets in Geophysics, Academic, San Diego, 1994.

Ge, Z.: Significance tests for the wavelet power and the wavelet power spectrum, Ann. Geophys., 25, 2259-2269, 2007, http://www.ann-geophys.net/25/2259/2007/.

Ge, Z. and Liu, P. C.: A time-localized response of wave growth process under turbulent winds, Ann. Geophys., 25, 1253-1262, 2007, http://www.ann-geophys.net/25/1253/2007/.

Ge, Z. and Liu, P. C.: Long-term wave growth and its linear and nonlinear interactions with wind fluctuations, Ann. Geophys., 26, 747-758, 2008, http://www.ann-geophys.net/26/747/2008/.

Grinsted, A., Moore, J. C., and Jevrejeva, S.: Application of the cross wavelet transform and wavelet coherence to geophysical time series, Nonlin. Processes Geophys., 11, 561-566, 2004, http://www.nonlin-processes-geophys.net/11/561/2004/.

Jenkins, G. M. and Watts, D. G.: Spectral analysis and its applications, Holden-Day, San Francisco, 1968.

Kotz, S. and Srinivasan, R.: Distribution of product and quotient of Bessel function variates, Ann. I. Stat. Math., 21, 201-210, 1969.

Liu, P. C.: Wavelet spectrum analysis and ocean wind waves, Wavelets in Geophysics, edited by: Foufoula-Georgiou, E. and Kumar, P., Academic, San Diego, p. 151-166, 1994.

Lundstedt, H., Liszka, L., Lundin, R., and Muscheler, R.: Longterm solar activity explored with wavelet methods, Ann. Geophys., 24, 769-778, 2006, http://www.ann-geophys.net/24/769/2006/.

Papoulis, A.: Probability, random variables, and stochastic processes, McGraw-Hill, New York, 1965.

Rigozo, N. R., da Silva, H. E., Nordemann, D. J. R., Echer, E., Echer, M. P. D., and Prestes, A.: The medieval and modern maximum solar activity imprints in tree ring data from Chile and stable isotope records from Antarctica and Peru, J. Atmos. Sol.-Terr. Phys., 70, 1012-1024, 2008.

Torrence, C. and Compo, G. P.: A practical guide to wavelet analysis, B. Am. Meteorol. Soc., 79, 61-78, 1998.

Torrence, C. and Webster, P. J.: Interdecadal changes in the ENSOMonsoon system, J. Climate, 12, 2679-2690, 1999.

Van Milligen, B. Ph., Sánchez, E., Estrada, T., Hidalgo, C., Brañas, B., Carreras, B., and García, L.: Wavelet bicoherence: A new turbulence analysis tool, Phys. Plasmas, 2, 3017-3032, 1995.

Wells, W. T., Anderson, R. L., and Cell, J. W.: The distribution of the product of two central or non-central chi-square variates, Ann. Math. Stat., 33, 1016-1020, 1962. 